Research Article

\title{
Tuina Massage Improves Cognitive Functions of Hypoxic-Ischemic Neonatal Rats by Regulating Genome-Wide DNA Hydroxymethylation Levels
}

\author{
Yunpeng Zhang, ${ }^{1,2}$ Chao Gao, ${ }^{3}$ Danmei Chen, ${ }^{1,2}$ Cuiting Wang, ${ }^{1,2}$ Long Chen,,4 \\ Yaodong Zhang, ${ }^{5}$ and Bing $\mathrm{Li} \mathbb{C}^{1,2}$ \\ ${ }^{1}$ Research Center for Clinical Medicine, Jinshan Hospital, Fudan University, Shanghai, China \\ ${ }^{2}$ Institute of Neurology, Academy of Integrative Medicine, Fudan University, Shanghai, China \\ ${ }^{3}$ Department of Rehabilitation, Henan Children's Hospital, Zhengzhou University, Zhengzhou, China \\ ${ }^{4}$ Department of Neurology, Jinshan Hospital, Fudan University, Shanghai, China \\ ${ }^{5}$ Department of Pediatrics, Henan Children's Hospital, Zhengzhou University, Zhengzhou, China \\ Correspondence should be addressed to Bing Li; libingbm@163.com
}

Received 20 May 2019; Revised 5 September 2019; Accepted 30 September 2019; Published 23 October 2019

Academic Editor: Shan-Yu Su

Copyright (c) 2019 Yunpeng Zhang et al. This is an open access article distributed under the Creative Commons Attribution License, which permits unrestricted use, distribution, and reproduction in any medium, provided the original work is properly cited.

\begin{abstract}
In addition to abnormalities of motor and posture, children with cerebral palsy (CP) often have intellectual disability. As a complementary and alternative traditional Chinese medicine (TCM) therapy, Chinese Tuina massage, also called Tuina in China, has been widely applied in clinical treatment for CP in China for a long time. However, the molecular basis for this still remains largely unknown. Recently, DNA hydroxymethylation has been shown to be sensitive to environment and plays critical roles in some neurological disorders, whereas the research focusing on the relationship between $5 \mathrm{hmC}$ and Tuina therapy for cerebral palsy is deficient. In our study, we first observed that Tuina improved learning and memory functions of hypoxic-ischemic (HI) rat pups. Meanwhile, $5 \mathrm{hmC}$ level of the temporal lobe cortex in the HI neonatal rat model is decreased significantly compared to that of the rats in control and Tuina groups. Then, we used the hMeDIP-Seq method to explore whether and how DNA hydroxymethylation is involved in Tuina therapy for cerebral palsy. Genomic annotation of DhMRs of HI group's hypo-hydroxymethylation to genes revealed enrichment in multiple neurodevelopmental signaling pathways. Moreover, we found the depletion of $5 \mathrm{hmC}$ modifications in genes associated with neuronal development was accompanied by reduced mRNA levels of these genes. Taken together, our results indicate that Tuina may regulate the expression of neurodevelopment-related genes by changing the status of DNA hydroxymethylation, thereby improving learning and memory functions of cerebral palsy.
\end{abstract}

\section{Introduction}

Cerebral injury occurring during the perinatal period is a major cause for acquired disability. The occurrence of cerebral injury in infants is correlated with multiple factors, such as hypoxia-ischemia, infection, oxidative stress, and inflammatory response $[1,2]$ among which hypoxia-ischemia (HI) may induce cerebral palsy $(\mathrm{CP})$ by triggering perinatal cerebral lesions in preterm [3]. CP is the commonest neonatal chronic disability, characterized by motor and postural impairments, and is often accompanied by cognitive and learning deficits [4]. In basic science studies, the HI neonatal rat model has been widely used in exploring the behavioral outcomes and pathogenesis of cerebral palsy [5-7]. Despite the great advancement in CP studies, the molecular mechanisms of how hypoxia-ischemia contributes to $\mathrm{CP}$ development and progression still stay unclear.

To this day, the current therapies for $\mathrm{CP}$ mainly includes orthopedic surgery, antispasticity medications, and various kinds of motor learning interventions [8], most of which 
merely work to treat the complications related to $\mathrm{CP}$ and may bring about side effects. However, traditional Chinese medicine (TCM), including Tuina, acupuncture, and herbal medicine, alone or combined with conventional therapy of western medicine, has been widely used as an alternative treatment for CP in China [9]. Tuina is an ancient form of medical massage in Chinese medicine, with applied finger pressure to acupoints that are putatively sensitized by organ impairment [10]. Notably, Tuina, as a safer and more effective intervention, has displayed obvious effects on cerebral palsy in clinical practice. However, the exact mechanism of how Tuina works on CP has not yet been elucidated.

On the contrary, pathogenically, environment-sensitive epigenetic modifications have already been proven to be involved in neurogenesis [11], learning and memory [12], and synaptic plasticity [13]. Among various epigenetic modifications, DNA methylation on the fifth carbon of cytosine (5-methylcytosine, $5 \mathrm{mC}$ ) is the most extensively studied epigenetic modification which plays important roles in chromatin structure remolding, transcriptional suppression, and cellular differentiation [14-16]. Furthermore, oxidation of $5 \mathrm{mC}$ into 5-hydroxymethylcytosine $(5 \mathrm{hmC})$ by the ten-eleven translocation (TET) gene family is proposed as a novel mechanism for removal of $5 \mathrm{mC}[17,18]$, and $5 \mathrm{hmC}$ has been suggested to be critical in maintenance of neuron structure and functions due to its obvious enrichment in the brain [19-21]. Meanwhile, $5 \mathrm{hmC}$ alteration is also identified by many studies to be associated with neurological disorders, such as Alzheimer's disease [22], Rett syndrome [23], fragile $\mathrm{X}$-associated tremor/ataxia syndrome [24], Huntington's disease [25], and the autism spectrum disorders [26]. These evidences provided the basis for the hypothesis that $5 \mathrm{hmC}$ alterations may play critical roles in nervous system disease.

To explore the presence of the involvement of Tuina therapy in the alteration of $5 \mathrm{hmC}$ in $\mathrm{CP}$, we applied an established chemical labeling and affinity purification method coupled with high-throughput sequencing technology to reveal the possible genome-wide $5 \mathrm{hmC}$ alterations in $\mathrm{HI}$ rats prior to and after Tuina treatment in order to seek the relationship between $5 \mathrm{hmC}$ and Tuina therapeutic effects on $\mathrm{CP}$, thereby providing evidence to support application of Tuina therapy for cerebral palsy.

\section{Materials and Methods}

2.1. Animal Model. All experiments were approved by the Ethics Committee for Animal Care of Jinshan Hospital affiliated to Fudan University. A modified Rice-Vannucci model was used as previously described $[27,28]$. The neonatal HI model was performed on Sprague-Dawley male rat pups (Slac Laboratory Animal Company, Shanghai, China) on postnatal day 3 (P3) via ligation of the left common carotid artery under $3 \%$ isoflurane anesthesia. Surgery time for each rat was controlled within 10 minutes. After recovery for 1 hour, these pups were placed in a hypoxia chamber at $37^{\circ} \mathrm{C}$ and subject to the gas mixer composed of $8 \% \mathrm{O}_{2}$ and $98 \% \mathrm{~N}_{2}$ for 3.5 hours. Sham animals had the same operation to expose the common carotid artery without ligation and hypoxia. After surgery, the rat pups were returned to their home cage.

2.2. Tuina Treatment. Tuina treatment was performed by a specialized researcher with professional physician certificate. From the day of $\mathrm{P} 4$ to $\mathrm{P} 31$, rat pups in the Tuina group accepted Tuina intervention which is carried out 120 times/ min for 15 minutes, once a day. The procedure of Tuina is as follows: first, the rat was placed on the palm of the manipulator's left hand to adjust to the environment; secondly, the manipulator used the middle finger of his right hand to gently rub the rat from the neck to tail three times to relax its muscles; thirdly, Tuina was carried out by successive rubbing, kneading, and one-finger pushing along the spine of the rats from top to bottom and from inside out on dorsum and ambilateral ligaments as well as the muscles along the du meridian and bladder meridian of the rat with reference to related traditional Chinese medicine (TCM) works.

2.3. Righting Reflex. Righting reflex of neonatal rats was assessed from P5 to P11, as described before [29, 30]. During this test, the rat pups were placed in a supine position on a cleaned flat surface and the time for these pups to reachieve the prone position with all four paws was measured.

2.4. Gait Reflex. Rat pups were placed in the center of a white rounded cardboard $(13 \mathrm{~cm}$ in diameter), and the day when they moved off the circle with both forelimbs was recorded [31].

2.5. Inclined Plane Test. This test was used to measure limb strength by recording the maximal angle of the slope onto which the rat pups can still manage to grip on the day of P21, as shown in Figure 1(b) [32]. During this test, the rats were placed facing right or left and the angle of the plane was increased by $5^{\circ}$ every five seconds in order to determine the maximal angle of the slope to which the rat could still hold [33].

2.6. Step-Down Avoidance Test. This test was implemented to measure the retention of memory $[34,35]$. A rat was gently placed on a cylindrical insulation platform $(4.5 \mathrm{~cm}$ in diameter and height) in a conditioning chamber to adapt to the surrounding environment for $3 \mathrm{~min}$. Once the rat steps down the platform, it will be electrified immediately. In the 5-minute-training session (P26), the rats were trained to jump back onto the platform to avoid the electric stimulation. In the test sessions ( $24 \mathrm{~h}$ after training, i.e., P27), the latency time (the time for the rats to step down from the platform for the first time) and the number of errors (frequency of the rats stepping down from the platform) were recorded.

2.7. Morris Water Maze (MWM) Test. In order to evaluate spatial learning and memory, the Morris water maze test was performed for 5 days as previously described [36, 37]. The 


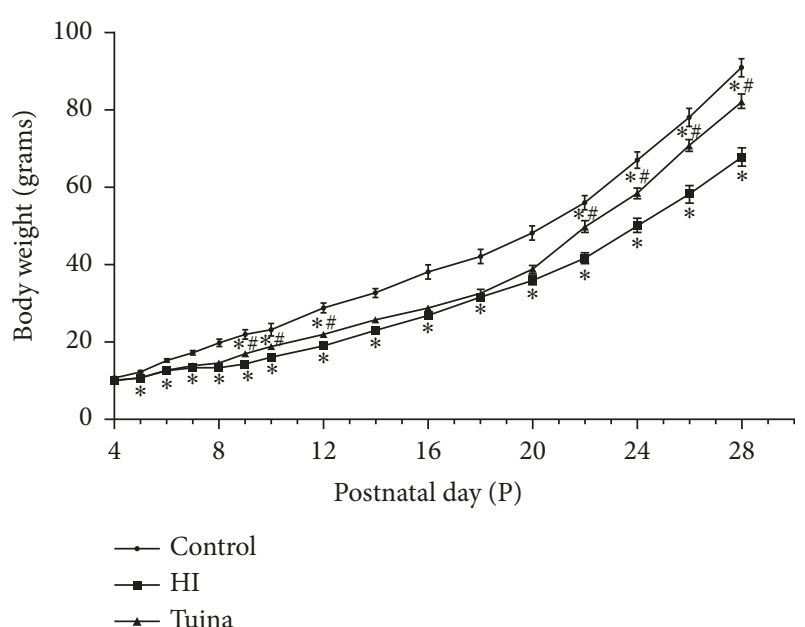

(a)

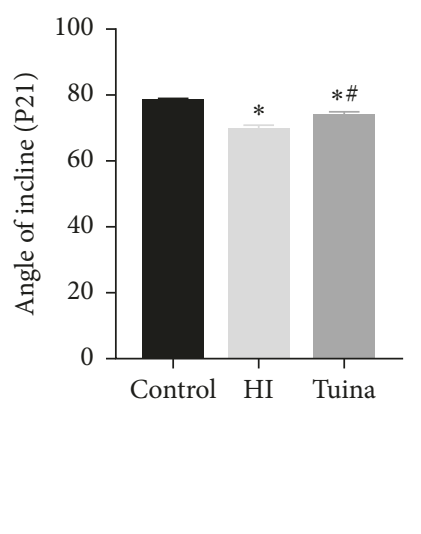

(b)

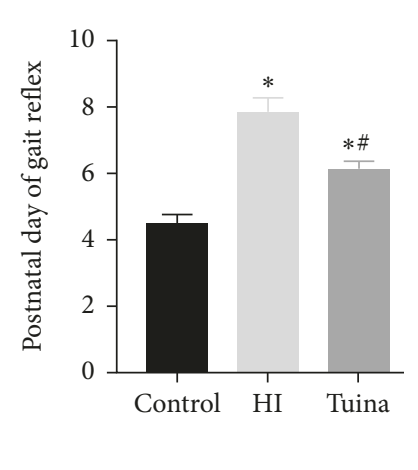

(c)

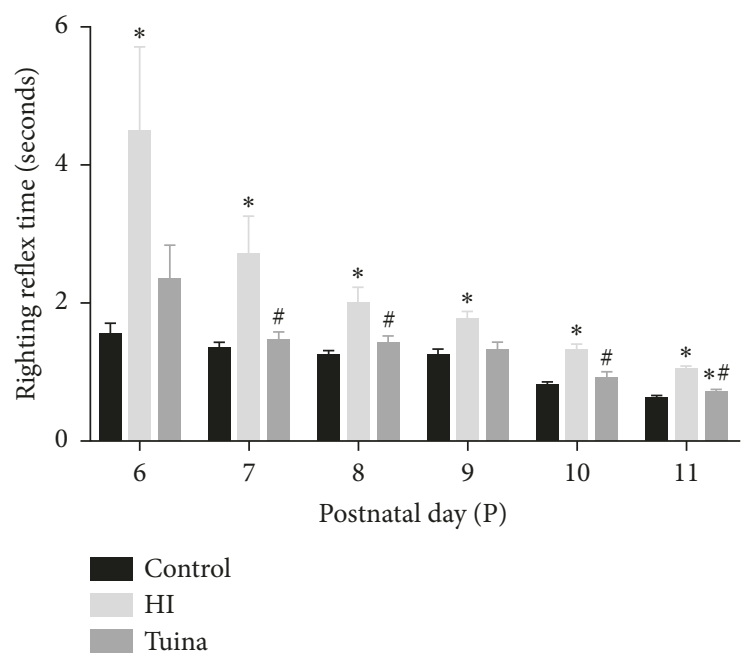

(d)

Figure 1: Tuina improves hypoxia-ischemia-induced weight loss, limb strength weakness, and neurological reflexes disorder: (a) average weights of rat pups $(n=11)$ starting from the 4th day after birth (P4) (i.e., 1 day after the hypoxic-ischemic insult); (b) angle of the inclined plane test on P21 $(n=11)$; (c) the postnatal day of gait reflex $(n=11)$; (d) righting reflex time $(n=11)\left({ }^{*}\right.$ vs. control group, $p<0.05$; ${ }^{\#}$ vs. HI group, $p<0.05)$.

water maze (Shanghai Xinyuan Information Technology Company, Shanghai, China) was divided into four quadrants, and a platform $(5 \mathrm{~cm}$ in diameter) was placed $1 \mathrm{~cm}$ below the water surface in one quadrant. In the training sessions (P27-P30), all the rats were trained four times a day for four consecutive days, and they were allowed to find the hidden platform within $60 \mathrm{~s}$. If the rat failed to find the platform within $60 \mathrm{~s}$, it was gently guided to the platform and allowed to stay on it for $30 \mathrm{~s}$ before being sent back to the cage. On the day for the spatial probe trial (P31), the platform was removed and the rats were released into the water facing the pool wall from the same position as in the training sessions. Subsequently, the motion tracks and the number of platform location crossings were recorded.

2.8. Dot Blotting. After completion of the above tests, the rat pups were sacrificed on P31 and their brains were dissected for collecting the total DNA of the left temporal cortex by a QIAamp DNA Mini Kit (Qiagen, Germany). $2.5 \mu \mathrm{L}$ genomic DNA $(100 \mathrm{ng} / \mu \mathrm{L})$ was spotted on nylon membranes followed by baking at $80^{\circ} \mathrm{C}$ for $30 \mathrm{~min}$ to cross-link DNA [22]. After blocking with $5 \%$ nonfat milk for $1 \mathrm{~h}$ at room temperature, the membrane was incubated with polyclonal $5 \mathrm{hmC}$ antibody ( $1: 1000$ dilution, Active Motif, United States, Cat. No. 39769) at $4^{\circ} \mathrm{C}$ overnight and the horseradish peroxidase (HRP)-conjugated secondary antibody was used to probe the next day. The dot blot membrane was stained with $0.02 \%$ methylene blue (MB) to ensure equal loading.

2.9. Hydroxymethylated DNA Immunoprecipitation (hMeDIP) Analysis and High-Throughput Sequencing. Genomic DNA was extracted from the left temporal cortex of rat pups (control: $n=3$; HI: $n=3$; Tuina: $n=3$ ) and sonicated to 200-350 base pairs (bp). And the DNA fragments were end- 
repaired; A-tailed and Illumina adapters were ligated. Then, adapter-ligated DNA was denatured and incubated with $5 \mathrm{hmC}$ antibody (Active Motif, United States, Cat. No. 39769) at $4^{\circ} \mathrm{C}$ overnight. Antibody-DNA complexes were captured by protein A/G beads. The immunoprecipitated DNA was purified and underwent high-throughput sequencing performed on the Illumina HiSeq3000 system (Illumina, United States) [38].

\subsection{Mapping of Sequencing Data and Bioinformatics.} Processing of sequencing data was performed as described previously [39]. FASTQ sequence files after biological duplications were aligned to UCSC rat reference genome (RGSC6.0/rn6) [40]. Unique, nonduplicate reads were used for peak calling and annotation by hypergeometric optimization of motif enrichment (HOMER) [41]. 5-hmC enrichment peaks were determined using a model-based analysis of ChIP-Seq (MACS) software [42]. And differentially hydroxymethylated regions (DhMRs) were identified among all pairs of 5 -hmC-enriched samples by directly comparing one sample with another in every direction [43]. Gene ontology (GO) analyses were performed using the DAVID [44].

2.11. Quantitative RT-PCR. Total RNA was extracted from the left temporal lobe cortex. Reverse transcription of cDNA was performed using the PrimeScript RT Master Mix Kit (Takara, Japan). Quantitative RT-PCR reactions were performed on 7500 real-time PCR system (Applied Biosystems, United States) using SYBR green (Takara, Japan). $\beta$-actin was used as an internal control, and relative expression of target gene was determined by the $2^{-\Delta \Delta C T}$ method. The sequences of primers used in the present experiment were listed as follows: Prkg2 primer (forward 5'-CCTCTGGATGTTCACCGCAAGAC-3', reverse $5^{\prime}$-AGGTCATAGGTCCGGCTTGTGG-3'), Casd1 primer (forward 5'-GAGAGCAGACGGATGAATGGAAGG-3' , reverse $5^{\prime}$-AACAGATAAGCAGCCACCAGAACG-3'), Slc44a1 primer (forward $5^{\prime}$-ACACAGCCACAGCCATCAATAGC-3', reverse $5^{\prime}$-CAGCCACTCGCAGAGCATTCTC- $3^{\prime}$ ), and $\beta$-actin primer (forward $5^{\prime}$-CATGTACGTTGCTATCCAGGC- $3^{\prime}$, reverse $5^{\prime}$-CTCCTTAATGTCACGCACGAT-3').

2.12. Western Blotting Assay. The isolated left temporal lobe cortex was added with ice-cold protein lysate and centrifuged at $12000 \mathrm{r} / \mathrm{min}$ for $20 \mathrm{~min}$ at $4^{\circ} \mathrm{C}$. Protein samples were separated by electrophoresis using SDS-PAGE and transferred onto PVDF membranes (Millipore, United States). After blocking with $5 \%$ nonfat milk for $2 \mathrm{~h}$ at room temperature, the membranes were incubated with the primary antibodies at $4^{\circ} \mathrm{C}$ overnight and then incubated with HRPconjugated secondary IgG antibodies. The primary antibodies used here included anti-Tet1 (1:1000, Abcam, UK, Cat. No. 191698), anti-Tet2 (1:1000, Millipore, United States, Cat. \# ABE364), anti-Slc44a1 (1:1000, Abcam, UK, Cat. No. 110767), and anti- $\beta$ tubulin $(1: 2000$, Cell Signaling Technology, United States, Cat. \#2128). The membranes were then developed using ECL detection, and the signals were detected with Tanon-5200 Chemiluminescent Imaging System (Tanon, Shanghai, China). The protein levels were analyzed by Image J software (NIH, Bethesda, USA) and normalized to the corresponding $\beta$-tubulin level.

2.13. Immunofluorescence Confocal Microscopy. Rat pups were sacrificed on P31, and their hearts were perfused with $0.9 \%$ saline followed by $4 \%$ paraformaldehyde (PFA). After perfusion, the brains were dissected and fixed in $4 \%$ PFA overnight followed by being embedded in paraffin and made into $5 \mu \mathrm{m}$ serial sections. After microwave antigen retrieval in citric acid buffer at $95^{\circ} \mathrm{C}$, nonspecific sites were blocked with $5 \%$ bovine serum albumin (BSA) for $1 \mathrm{~h}$ at room temperature. Next, the brain sections were incubated with $5 \mathrm{hmC}$ primary antibody ( $1: 1000$, Active Motif, United States, Cat. No. 39769) overnight at $4^{\circ} \mathrm{C}$ followed by incubation with the secondary antibody (1:200, Life Technology, United States) $1 \mathrm{~h}$ at $37^{\circ} \mathrm{C} .4^{\prime}, 6$-diamino-2-phenylindole (DAPI) was used for nuclear immunofluorescence staining, and the immunofluorescence pictures were visualized and captured on a confocal microscope (Leica sp5, Germany).

2.14. Statistical Analysis. All the data were expressed as the mean \pm standard error of the mean (SEM) and analyzed by using SPSS. One-way analysis of variance (ANOVA) was applied to determine the differences among the 3 groups, followed by nonparametric $t$-test and Tukey honestly significant difference test. $P$ value $<0.05$ was considered statistically significant.

\section{Results}

Tuina improved weight loss, weakness of limb strength, and disorders of neurological reflexes induced by $\mathrm{HI}$.

Body weight of rat pups was measured from P4 to P28, and it was found that, from P22 to P28, the body weight of the rats in the Tuina group $(n=11)$ increased significantly compared to the HI group ( $n=11$; Figure $1(\mathrm{a})$ ). The results of the inclined plane test showed that the maximum angles were higher in the Tuina group than those in the HI group (Figure 1(b)), which indicated improvement in the weakness of limb strength in the Tuina group. The results of nervous system reflexes were as the following: in the gait reflexes test, the rat pups in the HI group started to move away from the circle with both forelimbs significantly later than those in control and Tuina groups (Figure 1(c)). In the righting reflex test, the rats in the Tuina group took shorter time to right themselves than those in the HI group from P7 to P11 (Figure 1(d)).

Tuina promoted learning and memory functions of hypoxic-ischemic rat pups.

Morris water maze test and step-down test were performed to evaluate the learning and memory functions of the rats. As shown in Figure 2(a), the motion tracks on the probe trial day of the MWM test show that the number of platform location crossings of the HI group was significantly less than that of the control group and Tuina group (Figure 2(b)). In the step-down test, the HI rats showed significantly shorter 

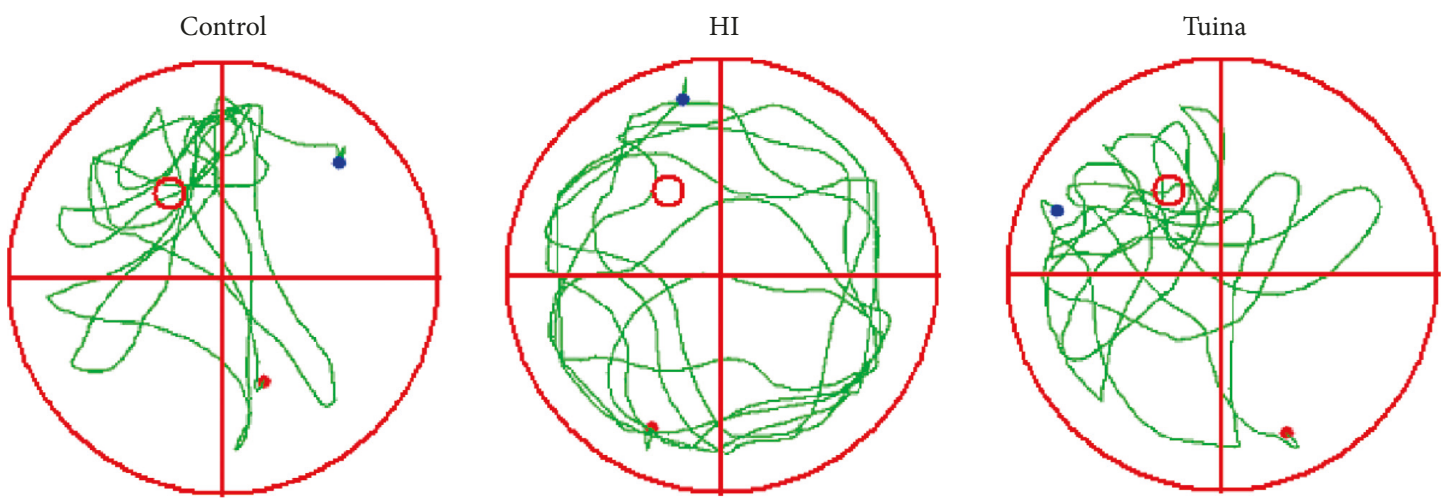

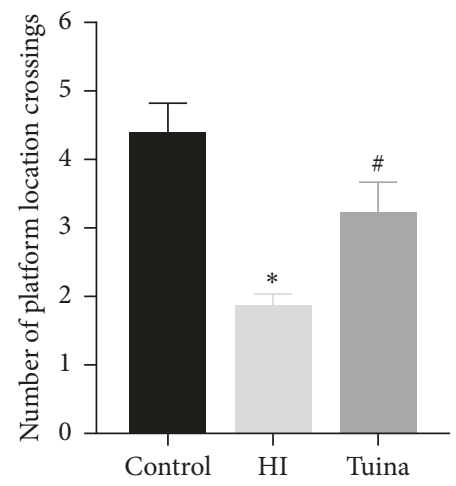

(b)

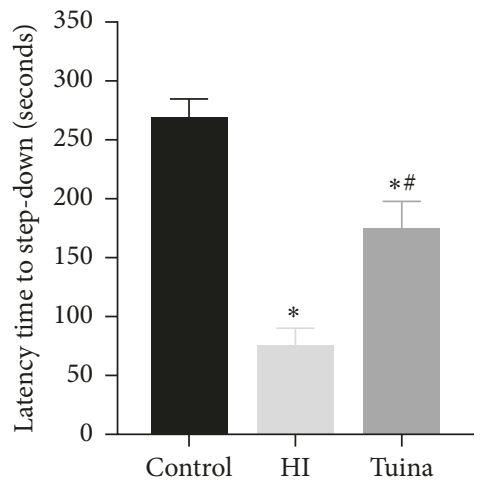

(c)

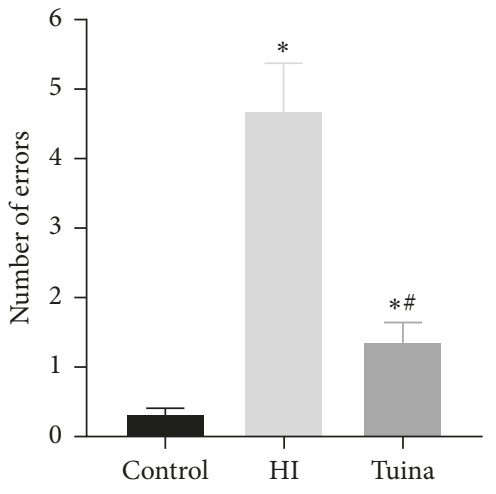

(d)

FIgURE 2: Tuina improves the learning and memory function in neonatal HI rats: (a) representative swimming paths of three groups $(n=11)$; (b) the average numbers of platform location crossings during the probe trial of the MWM test $(n=11)$; (c) the latency time of the step-down test $(n=11)$; (d) the errors number of the step-down test $(n=11)\left({ }^{*}\right.$ vs. control group, $p<0.05 ;{ }^{*}$ vs. HI group, $\left.p<0.05\right)$.

latency time and more errors compared to control and Tuina rats (Figures 2(c) and 2(d)).

Tuina increased $5 \mathrm{hmC}$ and Tet2 levels in the cerebral temporal cortex of HI rats.

To examine whether the level of $5 \mathrm{hmC}$ was affected during $\mathrm{CP}$ development, we performed dot blot tests and observed that the $5 \mathrm{hmC}$ level of $\mathrm{HI}$ rats decreased significantly compared to that of control, while this level was partially increased after Tuina $(P<0.05$; Figure 3(a)). Meanwhile, immunofluorescence staining demonstrated the merging of $5 \mathrm{hmC}$ and DAPI staining and $\mathrm{HI}$ led to decrease in genomic $5 \mathrm{hmC}$ in the left temporal cortex, while Tuina treatment restored $5 \mathrm{hmC}$ level to some degree (Figure 3(b)), which was in consistency with the results of dot blotting. Moreover, as shown in Figures 3(c) and 3(d), the HI group displayed decreased Tet 1 and Tet2 expression compared to the control group, whereas only Tet 2 was increased after Tuina therapy.

3.1. Genome-Wide $5 \mathrm{hmc}$ Alteration and Distribution. To investigate the exact location and distribution of genomewide $5 \mathrm{hmc}$, hydroxymethylated DNA immunoprecipitation (hMeDIP) followed by next-generation sequencing (hMeDIP-seq) [23] was carried out. The rats, respectively, from Control $(n=3)$, HI $(n=3)$, and Tuina $(n=3)$ groups were sacrificed on P31, and genomic DNA of the left temporal lobe cortex was extracted for hMeDIP-seq. Genome-wide pattern of $5 \mathrm{hmC}$ level was evaluated by counting $5 \mathrm{hmC}$ mapped reads in every $10 \mathrm{~kb}$ bin from the samples of the control, Tuina, and HI groups and then normalized to the total sequencing coverage [24]. As shown in Figures 4(a)-4(c), the distribution of the genome-wide $5 \mathrm{hmC}$ reads in the three groups was different, and then we further explored the specific gene regions in which these differences exist. Figure 4(d) shows the overlapping features of normalized density of $5 \mathrm{hmC}$ reads with the known genomic features on defined gene bodies and CGI (CpG islands) according to the UCSC table for RGSC6.0/rn6. Furthermore, as shown in Figure 4(e), the distributions of $5 \mathrm{hmC}$ at gene bodies and $1 \mathrm{~kb}$ up- and downstream of gene bodies were also studied. It can be observed from Figures 4(d) and 4(e) that the $5 \mathrm{hmC}$ level of the HI group differed from those of control group and Tuina group at the $5^{\prime}$ end of the genomic body, especially at the sites of $\pm 500 \mathrm{bp}$ of the transcriptional start sites (TSS) and the CGI within $500 \mathrm{bp}$ of TSS.

3.2. Identification and Annotation of Differentially Hydroxymethylated Regions (DhMRs). To pinpoint the specific loci exhibiting altered $5 \mathrm{hmC}$ profiles, we proceeded to identify and characterize the specific DhMRs of all autosomes and sex chromosomes across the genome. Interestingly, there was great difference in the distribution of hyper-DhMRs 


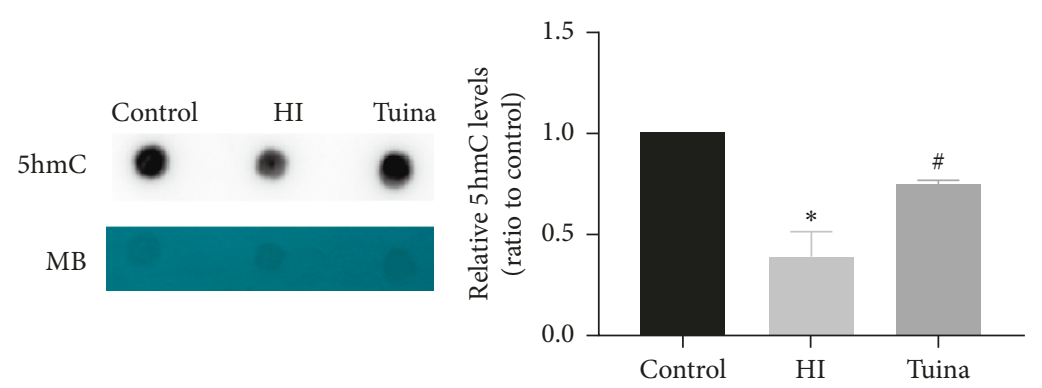

(a)

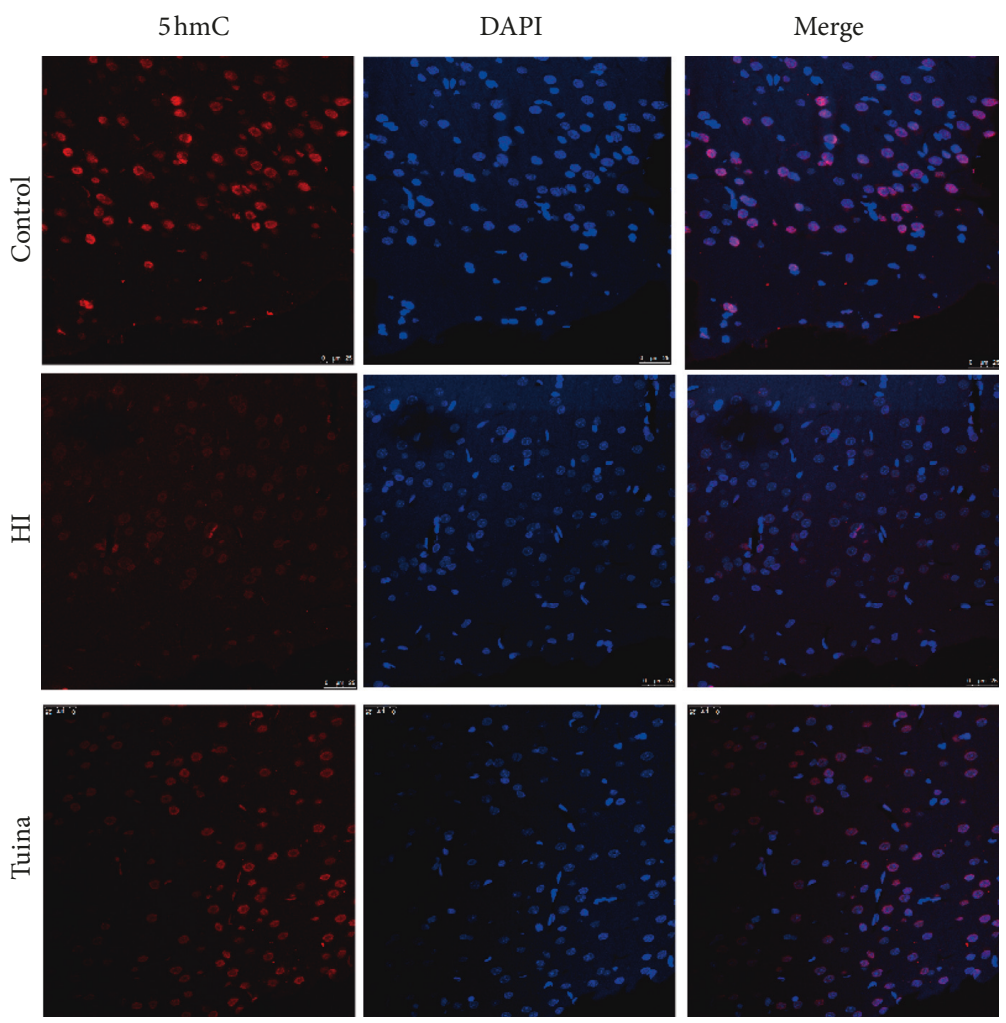

(b)
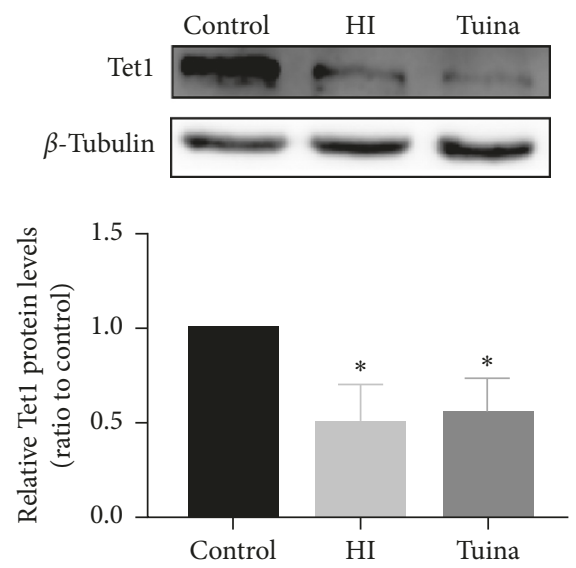

(c)

Figure 3: Continued. 


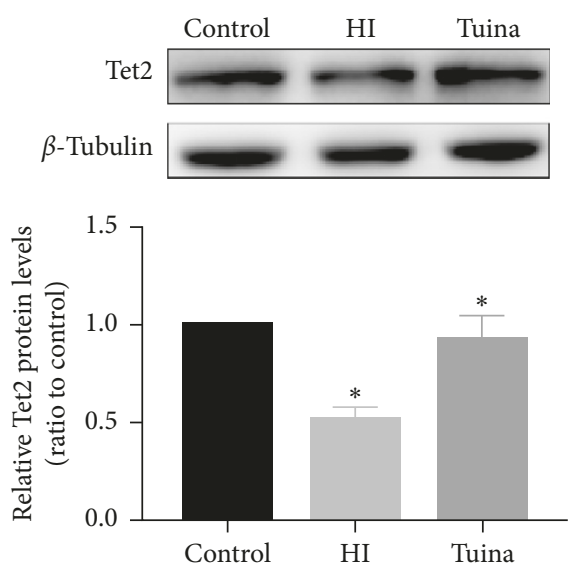

(d)

Figure 3: Reduced $5 \mathrm{hmC}$ and Tet proteins level in the HI rat cortex. (a) Dot blotting analysis of genomic $5 \mathrm{hmC}(n=5)$. MB staining was used as a loading control. (b) Immunofluorescence staining in the left temporal cortex. $5 \mathrm{hmC}$ was labeled with Alexa Fluor 594 (red), and the neuron nuclei were labeled with DAPI (blue) (Bars, $25 \mu \mathrm{m})$. Western blotting results of Tet1 (c) $(n=5)$ and Tet2 (d) $(n=5)$ protein ( ${ }^{*}$ vs. control group, $p<0.05 ;{ }^{\#}$ vs. HI group, $\left.p<0.05\right)$.

and hypo-DhMRs on the $\mathrm{X}$ chromosome (Figures 5(a)5(c)). To further explore the biological significance of the found DhMRs, the genes associated with these DhMRs were extracted for gene ontology (GO) enrichment analysis. As shown in Figures 5(d) and 5(e), GO enrichment analysis indicated that these hypo-DhMRs in the HI group were found highly enriched on the functional pathways related to neurodevelopment and neuronal functions, including developmental cell growth, neuron projection extension, positive regulation of developmental growth, developmental growth involved in morphogenesis, and glial cell differentiation.

Based on the comprehensive analysis of hydroxymethylation differential genes lists of the three groups, we selected six hypo-DhMRs associated genes from the HI group which were all associated with the functions of nervous system to investigate the relationship between $5 \mathrm{hmC}$ alterations and transcription levels of these genes. As what's expected, it was found out that, in accordance with the reduced $5 \mathrm{hmC}$ modification in Casd1, Prkg2, and Slc44a1, the corresponding mRNA levels were also significantly decreased in the HI group in comparison with those in control and Tuina groups (Figure 5(f)).

\section{Discussion}

With general safety and reputed efficacy, traditional Chinese medicine (TCM) is readily accepted by the general population all over the world. TCM has been widely applied in various pediatric diseases, such as Ewing's sarcoma [45], asthma [46], Graves' disease [47], and pediatric allergic disorders [47]. Meanwhile, there are also studies indicating that TCM therapies including Tuina, herbal medicines, and acupuncture are well-tolerated and have positive therapeutic effects on cerebral palsy $[9,48-51]$. Due to the promising clinical results and fewer side effects, Chinese Tuina massage has attracted growing interest in terms of the treatment for CP.
As a new epigenetic modification, $5 \mathrm{hmC}$ not only serves as an intermediate of DNA demethylation processes but also acts as a stable epigenetic marker during development of various diseases [21]. $5 \mathrm{hmC}$ is about tenfold more enriched in neurons than in other cells and its dynamic regulation is critical in postnatal neurodevelopment [20, 23, 43]. Moreover, the alteration of the global $5 \mathrm{hmC}$ level has been considered closely related to many neurodegenerative disorders and neurodevelopmental diseases [22-26].

However, to the best of our knowledge, few studies have been focused on the connection between DNA hydroxymethylation and cerebral palsy, which became the inspiration for our research. In our study, the results of dot blotting and immunofluorescence revealed that the global $5 \mathrm{hmC}$ level in the cerebral temporal lobe cortex was significantly decreased after $\mathrm{HI}$ injury, which is consistent with the reduced $5 \mathrm{hmC}$ level in the mouse kidney after ischemiareperfusion [52]. However, after Tuina treatment, $5 \mathrm{hmC}$ level in the Tuina group was higher than that in the $\mathrm{HI}$ group. Furthermore, it is known that $5 \mathrm{hmC}$ is produced via oxidation of $5 \mathrm{mC}$ by Tet proteins, based on which we detected the levels of Tet 1 and Tet 2 proteins and found that both levels were significantly decreased after HI injury, whereas only Tet 2 protein was increased after Tuina treatment. In all, these findings imply that the reduced Tet 1 and Tet2 expression may contribute to the decrease in $5 \mathrm{hmC}$ after HI injury and Tuina may increase $5 \mathrm{hmC}$ level via enhancing Tet2 expression.

On the contrary, CGI methylation in promoters was found critical in gene silencing and $5 \mathrm{hmC}$ may play a role in gene expression mediated by DNA demethylation $[53,54]$. In our study, the HI group displayed decreased enrichment of genome-wide $5 \mathrm{hmC}$ in CGI within $500 \mathrm{bp}$ of TSS compared to control and Tuina groups, whereas Tuina increased genome-wide distribution of $5 \mathrm{hmC}$. Meanwhile, in line with the previous findings that the enrichment of $5 \mathrm{hmC}$ in the gene body may has a strong correlation with the increased transcription of the concerned genes in neurons 


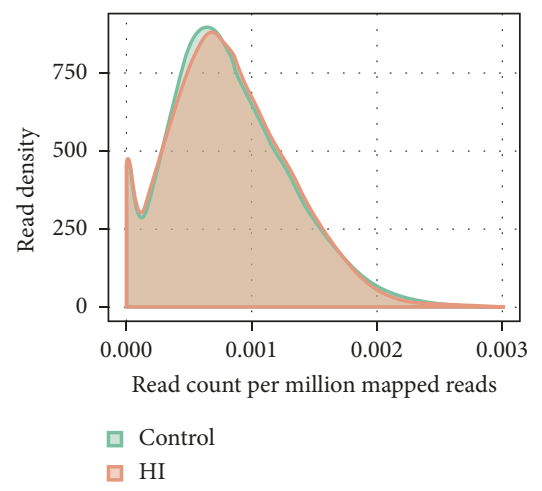

(a)

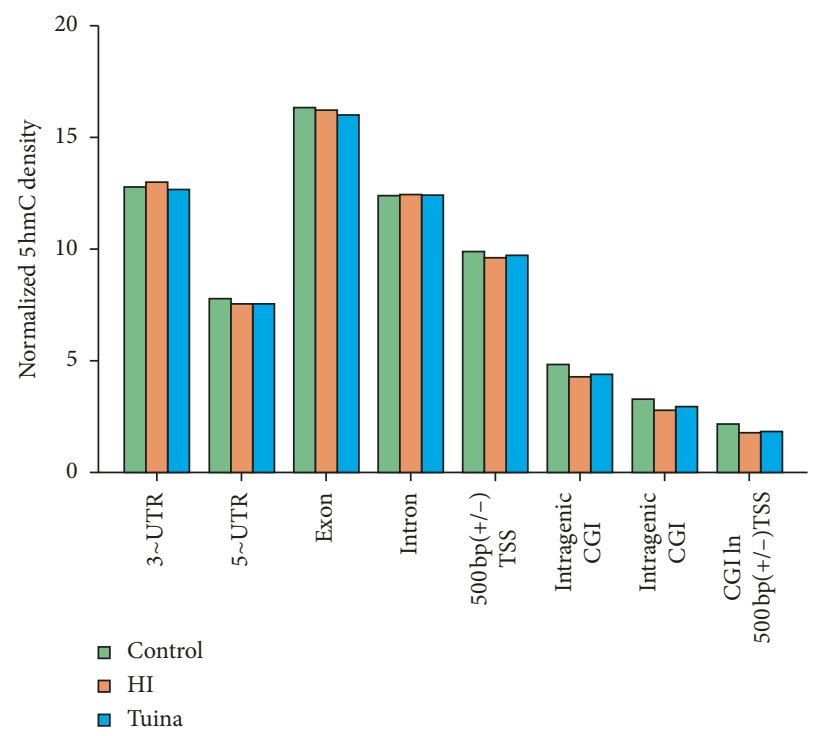

(d)

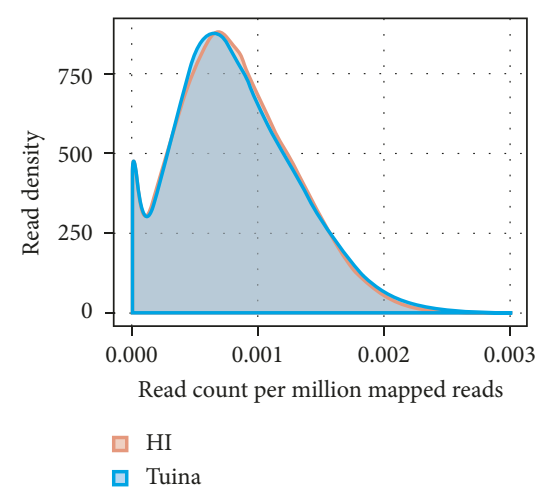

(b)

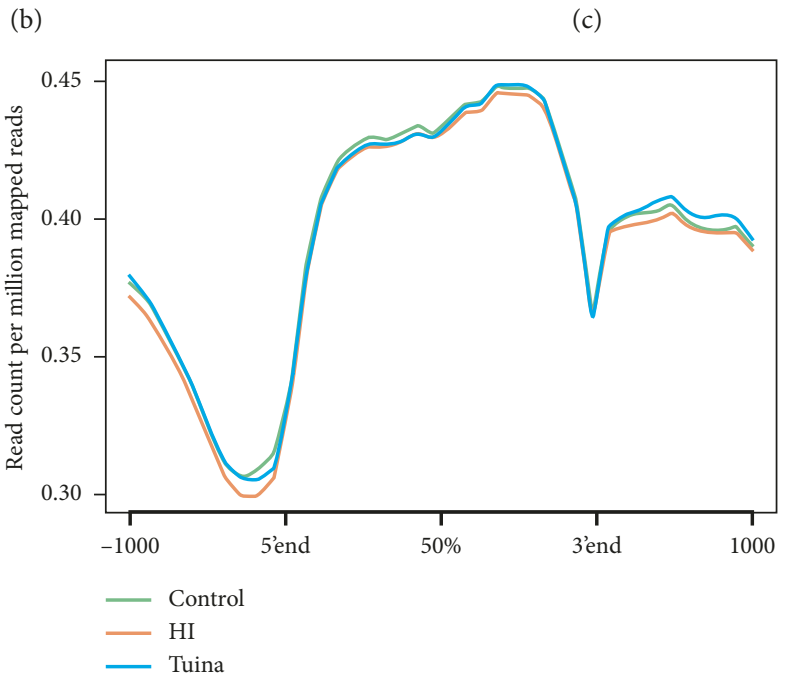

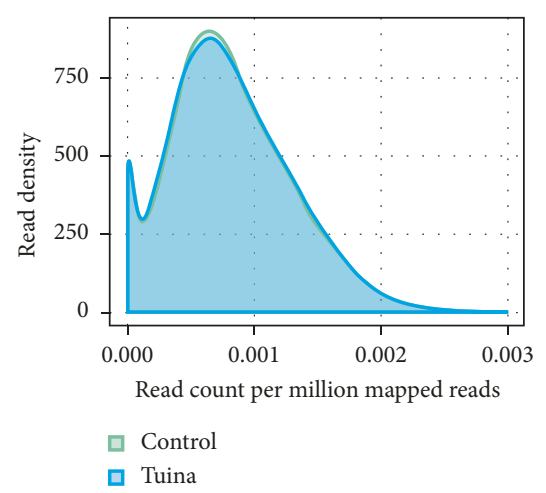

(c)

(e)

Figure 4: Genome-wide $5 \mathrm{hmc}$ alteration and distribution. (a-c) Genome-wide $5 \mathrm{hmC}$ reads density distribution. (d) Normalized 5 hmC densities overlapping with known genomic features. (e) Normalized $5 \mathrm{hmC}$ read densities across TSS, TES, and RefSeq gene bodies. Gene bodies were normalized to $0-100 \%$ as relative positions.

$[19,55]$, our RT-PCR results showed that the depletion of $5 \mathrm{hmC}$ modification in Prkg2, Casd1, and Slc44a1 gene was accompanied by the reduced mRNA levels of these genes in the HI group. Based on all these findings mentioned above, it was indicated that $5 \mathrm{hmC}$ modification may play a critical part in the pathogenesis of $\mathrm{CP}$ through regulating transcription and expression of the genes related to neuronal functions and Tuina may produce therapeutic effects on CP via modulating $5 \mathrm{hmC}$.

More interestingly, it was found that $\mathrm{CP}$ and related developmental disorders are more common seen in male patients $[56,57]$ and female neonatal mouse model of $\mathrm{HI}$ brain injury also displayed gender-specific lower occurrence of CP [58], the reason for which is still uncertain. In order to avoid the disruption from this possible genderspecific protective effects, only male rat pups were selected for our study. Remarkably, in genome-wide circular map (Figure 5(a)), great differences in the distribution of hyperDhMRs and hypo-DhMRs on the $\mathrm{X}$ chromosome were observed from the comparison between any two groups in our study, which further confirms the correlation between sex and the occurrence of cerebral palsy. Notably, due to the limitations of our lab, we could not do IgG control or generate the input libraries, and DhMRs were identified by directly comparing one to the other rather than comparing to the input, which we will intend to improve in our future studies to make the research more precise.

Furthermore, GO analyses of hypo-DhMRs-associated genes in the HI group showed that these genes are highly enriched in multiple signaling pathways related to neurodevelopment and neuronal functions. In our experiment, growth retardation and neurodevelopmental disorders appeared in the HI group in the form of weight loss, weakness in limb strength, and several neuronal reflex disorders which were improved by Tuina treatment to some extent. Moreover, from the results of the Morris water maze test and step-down avoidance test, Tuina improved learning and memory functions of hypoxic-ischemic rats significantly. These results indicate the correlation between the alteration in 5-hydroxymethylcytosine loci and neuronal development and furtherly 


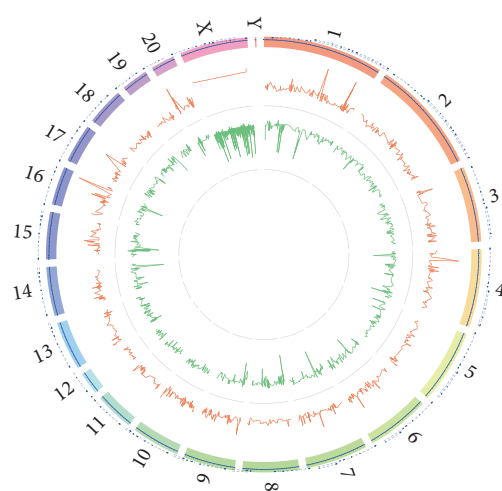

Control vs HI

- Hyper-DhMRs

— Hypo-DhMRs

(a)

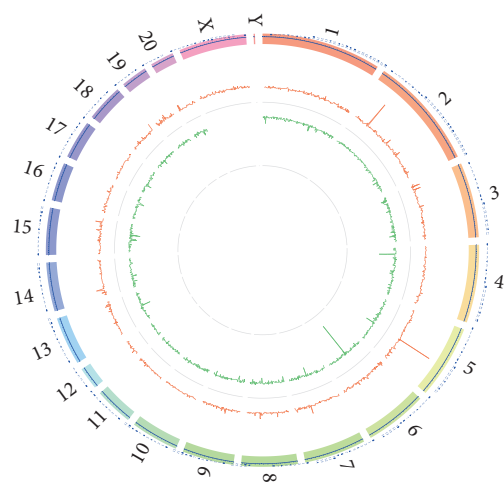

HI vs Tuina

- Hyper-DhMRs

— Hypo-DhMRs

(b)

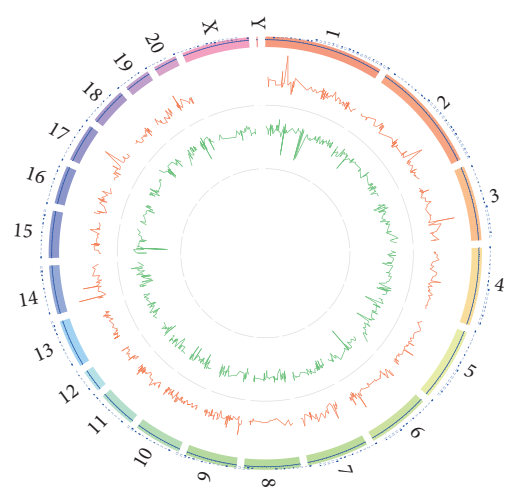

Control vs Tuina

- Hyper-DhMRs

— Hypo-DhMRs

Control vs HI Hypo-DhMRs GO analysis

Immune response-regulating cell surface receptor signaling pathway Cell surface receptor signaling pathway Contact inhibition Developmental cell growth Immune response-activating signal transduction Neuron projection extension Immune response-regulating signaling pathway Positive regulation of developmental growth Activation of immune response Developmental growth involved in morphogenesis -

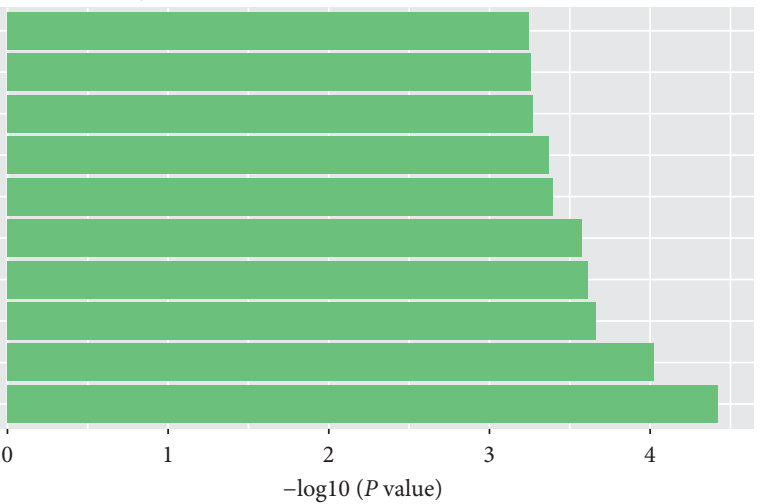

(d)

Tuina vs HI Hypo-DhMRs GO analysis

Regulation of transmembrane transport Activation of GTPase activity Regulation of ion transport Biological adhesion Glial cell differntiation Regulation of ion transmembrane transport Homophilic cell adhesion via plasma membrane adhesion molecules Localization -

Chemical homeostasis within a tissue Cell-cell adhesion via plasma-membrane adhesion molecules -

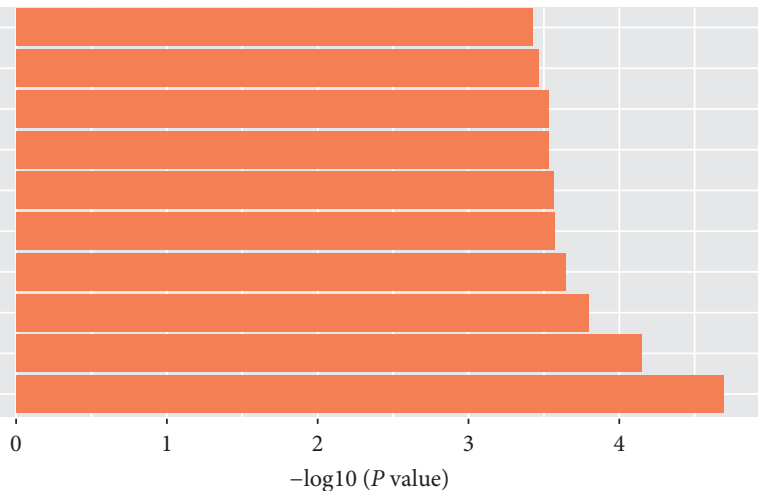

(e)
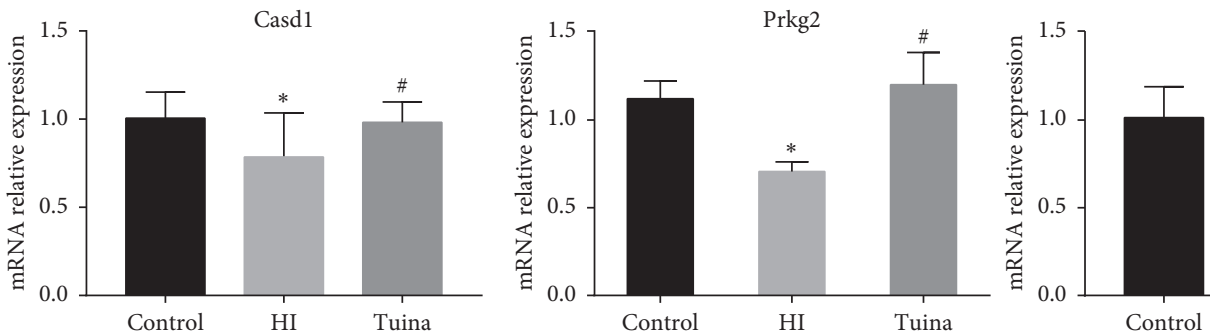

Slc44a1

(f)

FIGURE 5: Identification and characterization of differentially hydroxymethylated regions (DhMRs). (a-c) Chromosome circular map of genomewide DhMRs. Top ten GO enrichment analysis results of HI group's lower hydroxymethylation associated DhMRs compared to control (d) and Tuina (e) group. (f) RT-PCR analysis of the mRNA levels of selected hypo-DhMRs related genes. $\beta$-Actin was used as a control ( ${ }^{*}$ vs. control group, $p<0.05$; \# vs. HI group, $p<0.05)$. 
suggests that Tuina therapy may influence brain developments and cerebral cognitive functions via modulating DNA hydroxymethylation.

In the present study, we provided genome-wide $5 \mathrm{hmC}$ maps of control, HI, and Tuina groups and revealed alteration in DNA hydroxymethylation status and $5 \mathrm{hmC}$ dynamic change after Tuina treatment, which may shed light on the possible therapeutic mechanism of Tuina for CP.

\section{Data Availability}

The data used to support the findings of this study are available from the corresponding author upon request.

\section{Conflicts of Interest}

The authors declare that there are no conflicts of interest.

\section{Authors' Contributions}

Yunpeng Zhang and Chao Gao contributed equally to this work.

\section{Acknowledgments}

This work was supported by the National Natural Science Foundation of China (81774444), Henan Province Science and Technology Research Project (182102310403), ChinaCanada Cooperative Clinical Research Project, Henan Province Study Abroad Program (201721), and Henan Province Medical Science and Technology Research Project (Provincial and Ministry Construction Project) (201701034).

\section{References}

[1] N. Salmaso, B. Jablonska, J. Scafidi, F. M. Vaccarino, and V. Gallo, "Neurobiology of premature brain injury," Nature Neuroscience, vol. 17, no. 3, pp. 341-346, 2014.

[2] H. Hagberg, C. Mallard, D. M. Ferriero et al., "The role of inflammation in perinatal brain injury," Nature Reviews Neurology, vol. 11, no. 4, pp. 192-208, 2015.

[3] S. Marret, C. Vanhulle, and A. Laquerriere, "Pathophysiology of cerebral palsy," Handbook of Clinical Neurology, vol. 111, pp. 169-176, 2013.

[4] A. Colver, C. Fairhurst, and P. O. D. Pharoah, "Cerebral palsy," The Lancet, vol. 383, no. 9924, pp. 1240-1249, 2014.

[5] A. Stepanova, C. Konrad, S. Guerrero-Castillo et al., "Deactivation of mitochondrial complex I after hypoxia-ischemia in the immature brain," Journal of Cerebral Blood Flow \& Metabolism, vol. 39, no. 9, pp. 1790-1802, 2019.

[6] T. K. Kim, D. Park, Y. H. Ban et al., "Improvement by human oligodendrocyte progenitor cells of neurobehavioral disorders in an experimental model of neonatal periventricular leukomalacia," Cell Transplantation, vol. 27, no. 7, pp. 11681177, 2018.

[7] W. C. Tai, K. A. Burke, J. F. Dominguez, L. Gundamraj, and J. E. Turman Jr., "Growth deficits in a postnatal day 3 rat model of hypoxic-ischemic brain injury," Behavioural Brain Research, vol. 202, no. 1, pp. 40-49, 2009.

[8] I. Novak, S. McIntyre, C. Morgan et al., "A systematic review of interventions for children with cerebral palsy: state of the evidence," Developmental Medicine \& Child Neurology, vol. 55, no. 10, pp. 885-910, 2013.
[9] Y. Zhang, J. Liu, J. Wang, and Q. He, "Traditional Chinese Medicine for treatment of cerebral palsy in children: a systematic review of randomized clinical trials," The Journal of Alternative and Complementary Medicine, vol. 16, no. 4, pp. 375-395, 2010.

[10] S. Pritchard, Tui Na: A Manual of Chinese Massage Therapy, Churchill Livingstone, London, UK, 2010.

[11] Z. Wang, B. Tang, Y. He, and P. Jin, "DNA methylation dynamics in neurogenesis," Epigenomics, vol. 8, no. 3, pp. 401-414, 2016.

[12] N. K. Yu, S. H. Baek, and B. K. Kaang, "DNA methylationmediated control of learning and memory," Molecular Brain, vol. 4, no. 1, 5 pages, 2011.

[13] P. Munoz, C. Estay, P. Diaz, C. Elgueta, A. O. Ardiles, and P. A. Lizana, "Inhibition of DNA methylation impairs synaptic plasticity during an early time window in rats," Neural Plasticity, vol. 2016, Article ID 4783836, 13 pages, 2016.

[14] W. Reik, "Stability and flexibility of epigenetic gene regulation in mammalian development," Nature, vol. 447, no. 7143 , pp. 425-432, 2007.

[15] A. Zemach, I. E. McDaniel, P. Silva, and D. Zilberman, "Genome-wide evolutionary analysis of eukaryotic DNA methylation," Science, vol. 328, no. 5980, pp. 916-919, 2010.

[16] T. Jursch, C. Miskey, Z. Izsvak, and Z. Ivics, "Regulation of DNA transposition by $\mathrm{CpG}$ methylation and chromatin structure in human cells," Mobile DNA, vol. 4, no. 1, 15 pages, 2013.

[17] S. Kriaucionis and N. Heintz, "The nuclear DNA base 5hydroxymethylcytosine is present in Purkinje neurons and the brain," Science, vol. 324, no. 5929, pp. 929-930, 2009.

[18] M. Tahiliani, K. P. Koh, Y. Shen et al., "Conversion of 5methylcytosine to 5-hydroxymethylcytosine in mammalian DNA by MLL partner TET1," Science, vol. 324, no. 5929, pp. 930-935, 2009.

[19] M. A. Hahn, R. Qiu, X. Wu et al., "Dynamics of 5-hydroxymethylcytosine and chromatin marks in Mammalian neurogenesis," Cell Reports, vol. 3, no. 2, pp. 291-300, 2013.

[20] T. Khare, S. Pai, K. Koncevicius et al., " $5-\mathrm{hmC}$ in the brain is abundant in synaptic genes and shows differences at the exonintron boundary," Nature Structural \& Molecular Biology, vol. 19, no. 10, pp. 1037-1043, 2012.

[21] L. Tan and Y. G. Shi, "Tet family proteins and 5-hydroxymethylcytosine in development and disease," Development, vol. 139, no. 11, pp. 1895-1902, 2012.

[22] L. Shu, W. Sun, L. Li et al., "Genome-wide alteration of 5hydroxymenthylcytosine in a mouse model of Alzheimer's disease," BMC Genomics, vol. 17, no. 381, 2016.

[23] K. E. Szulwach, X. Li, Y. Li et al., "5-hmC-mediated epigenetic dynamics during postnatal neurodevelopment and aging," Nature Neuroscience, vol. 14, no. 12, pp. 1607-1616, 2011.

[24] B. Yao, L. Lin, R. C. Street et al., "Genome-wide alteration of 5-hydroxymethylcytosine in a mouse model of fragile X-associated tremor/ataxia syndrome," Human Molecular Genetics, vol. 23, no. 4, pp. 1095-1107, 2014.

[25] F. Wang, Y. Yang, X. Lin et al., "Genome-wide loss of 5-hmC is a novel epigenetic feature of Huntington's disease," Human Molecular Genetics, vol. 22, no. 18, pp. 3641-3653, 2013.

[26] L. A. Papale, Q. Zhang, S. Li, K. Chen, S. Keles, and R. S. Alisch, "Genome-wide disruption of 5-hydroxymethylcytosine in a mouse model of autism," Human Molecular Genetics, vol. 24, no. 24, pp. 7121-7131, 2015.

[27] J. E. Rice 3rd, R. C. Vannucci, and J. B. Brierley, "The influence of immaturity on hypoxic-ischemic brain damage in the rat," Annals of Neurology, vol. 9, no. 2, pp. 131-141, 1981.

[28] D. M. Doycheva, T. Hadley, L. Li, R. L. Applegate 2nd, J. H. Zhang, and J. Tang, "Anti-neutrophil antibody enhances 
the neuroprotective effects of G-CSF by decreasing number of neutrophils in hypoxic ischemic neonatal rat model," Neurobiology of Disease, vol. 69, pp. 192-199, 2014.

[29] S. Lien and J. D. Dickman, "Vestibular injury after low-intensity blast exposure," Frontiers in Neurology, vol. 9297 pages, 2018.

[30] J. Comhair, J. Devoght, G. Morelli et al., "Alpha2-Containing Glycine receptors promote neonatal spontaneous activity of striatal medium spiny neurons and support maturation of glutamatergic inputs," Frontiers in Molecular Neuroscience, vol. 11380 pages, 2018.

[31] A. Lubics, D. Reglodi, A. Tamas et al., "Neurological reflexes and early motor behavior in rats subjected to neonatal hypoxic-ischemic injury," Behavioural Brain Research, vol. 157, no. 1, pp. 157-165, 2005.

[32] S. Chen, J. Ye, X. Chen et al., "Valproic acid attenuates traumatic spinal cord injury-induced inflammation via STAT1 and NF-kappaB pathway dependent of HDAC3," Journal of Neuroinflammation, vol. 15, no. 1, 150 pages, 2018.

[33] T. M. Hallam, C. L. Floyd, M. M. Folkerts et al., "Comparison of behavioral deficits and acute neuronal degeneration in rat lateral fluid percussion and weight-drop brain injury models," Journal of Neurotrauma, vol. 21, no. 5, pp. 521-539, 2004.

[34] R. Lin, L. Li, Y. Zhang et al., "Electroacupuncture ameliorate learning and memory by improving $\mathrm{N}$-acetylaspartate and glutamate metabolism in APP/PS1 mice," Biological Research, vol. 51, no. 1, 21 pages, 2018.

[35] X. R. Han, X. Wen, Y. J. Wang et al., "Effects of CREB1 gene silencing on cognitive dysfunction by mediating PKA-CREB signaling pathway in mice with vascular dementia," Molecular Medicine, vol. 24, no. 1, 18 pages, 2018.

[36] M. Zhang, C. Qian, Z. G. Zheng et al., "Jujuboside A promotes Abeta clearance and ameliorates cognitive deficiency in Alzheimer's disease through activating Axl/HSP90/PPARgamma pathway," Theranostics, vol. 8, no. 15, pp. 4262-4278, 2018.

[37] R. Morris, "Developments of a water-maze procedure for studying spatial learning in the rat," Journal of Neuroscience Methods, vol. 11, no. 1, pp. 47-60, 1984.

[38] H. Zheng, X. Zhou, D. K. Li et al., "Genome-wide alteration in DNA hydroxymethylation in the sperm from bisphenol A-exposed men," PLoS One, vol. 12, no. 6, Article ID e0178535, 2017.

[39] R. Kim, K. L. Sheaffer, I. Choi, K. J. Won, and K. H. Kaestner, "Epigenetic regulation of intestinal stem cells by Tet1-mediated DNA hydroxymethylation," Genes \& Development, vol. 30, no. 21, pp. 2433-2442, 2016.

[40] B. Langmead, C. Trapnell, M. Pop, and S. L. Salzberg, "Ultrafast and memory-efficient alignment of short DNA sequences to the human genome," Genome Biology, vol. 10, no. 3, 2009.

[41] S. Heinz, C. Benner, N. Spann et al., "Simple combinations of lineage-determining transcription factors prime cis-regulatory elements required for macrophage and B cell identities," Molecular Cell, vol. 38, no. 4, pp. 576-589, 2010.

[42] Y. Zhang, T. Liu, C. A. Meyer et al., "Model-based analysis of ChIP-seq (MACS)," Genome Biology, vol. 9, no. 9, R137 pages, 2008.

[43] T. Wang, Q. Pan, L. Lin et al., "Genome-wide DNA hydroxymethylation changes are associated with neurodevelopmental genes in the developing human cerebellum," Human Molecular Genetics, vol. 21, no. 26, pp. 5500-5510, 2012.

[44] W. Huang da, B. T. Sherman, and R. A. Lempicki, "Systematic and integrative analysis of large gene lists using DAVID bioinformatics resources," Nature Protocols, vol. 4, no. 1, pp. 44-57, 2009.

[45] C. Ye, X. Yu, J. Zeng, M. Dai, and B. Zhang, "Effects of baicalein on proliferation, apoptosis, migration and invasion of Ewing's sarcoma cells," International Journal of Oncology, vol. 51, no. 6, pp. 1785-1792, 2017.

[46] H. Du, Y. Wang, Y. Shi, J. Yu, W. Sun, and Y. Zhang, "Effect of traditional Chinese medicine on inflammatory mediators in pediatric asthma," Mediators of Inflammation, vol. 2016, Article ID 5143703, 6 pages, 2016.

[47] Y. H. Luo, M. Zhu, D. G. Wang et al., "Yangxin Tongmai Formula ameliorates impaired glucose tolerance in children with Graves' disease through upregulation of the insulin receptor levels," Acta Pharmacologica Sinica, vol. 39, no. 6, pp. 923-929, 2018.

[48] W. L. Zhang, Y. A. Cao, J. Xia, L. Tian, L. Yang, and C. S. Peng, "Neuroprotective effect of tanshinone IIA weakens spastic cerebral palsy through inflammation, p38MAPK and VEGF in neonatal rats," Molecular Medicine Reports, vol. 17, no. 1, pp. 2012-2018, 2017.

[49] J. Jia, X. Shi, X. Jing et al., "BCL6 mediates the effects of Gastrodin on promoting M2-like macrophage polarization and protecting against oxidative stress-induced apoptosis and cell death in macrophages," Biophysical Research Communications, vol. 486, no. 2, pp. 458-464, 2017.

[50] L. M. Silva, M. Schalock, J. Garberg, and C. L. Smith, "Qigong massage for motor skills in young children with cerebral palsy and Down syndrome," American Journal of Occupational Therapy, vol. 66, no. 3, pp. 348-355, 2012.

[51] Y. Wu, L. P. Zou, T. L. Han et al., "Randomized controlled trial of traditional Chinese medicine (acupuncture and tuina) in cerebral palsy: part 1--any increase in seizure in integrated acupuncture and rehabilitation group versus rehabilitation group?," The Journal of Alternative and Complementary Medicine, vol. 14, no. 8, pp. 1005-1009, 2008.

[52] N. Huang, L. Tan, Z. Xue, J. Cang, and H. Wang, "Reduction of DNA hydroxymethylation in the mouse kidney insulted by ischemia reperfusion," Biochemical and Biophysical Research Communications, vol. 422, no. 4, pp. 697-702, 2012.

[53] A. M. Deaton and A. Bird, "CpG islands and the regulation of transcription," Genes \& Development, vol. 25, no. 10, pp. 1010-1022, 2011.

[54] X. Li, Y. Liu, T. Salz, K. D. Hansen, and A. Feinberg, "Wholegenome analysis of the methylome and hydroxymethylome in normal and malignant lung and liver," Genome Research, vol. 26, no. 12, pp. 1730-1741, 2016.

[55] B. M. Colquitt, W. E. Allen, G. Barnea, and S. Lomvardas, "Alteration of genic 5-hydroxymethylcytosine patterning in olfactory neurons correlates with changes in gene expression and cell identity," Proceedings of the National Academy of Sciences, vol. 110, no. 36, pp. 14682-14687, 2013.

[56] S. M. Reid, E. Meehan, C. S. Gibson et al., "Biological sex and the risk of cerebral palsy in victoria, Australia," Developmental Medicine \& Child Neurology, vol. 58, no. 2, pp. 43-49, 2016.

[57] M. V. Johnston and H. Hagberg, "Sex and the pathogenesis of cerebral palsy," Developmental Medicine \& Child Neurology, vol. 49, no. 1, pp. 74-78, 2006.

[58] P. M. Pimentel-Coelho, J. P. Michaud, and S. Rivest, "Evidence for a gender-specific protective role of innate immune receptors in a model of perinatal brain injury," Journal of Neuroscience, vol. 33, no. 28, pp. 11556-11572, 2013. 


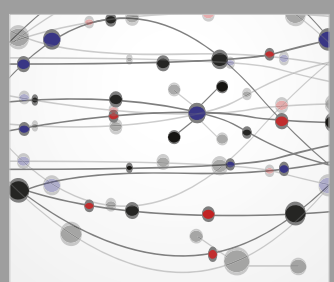

The Scientific World Journal
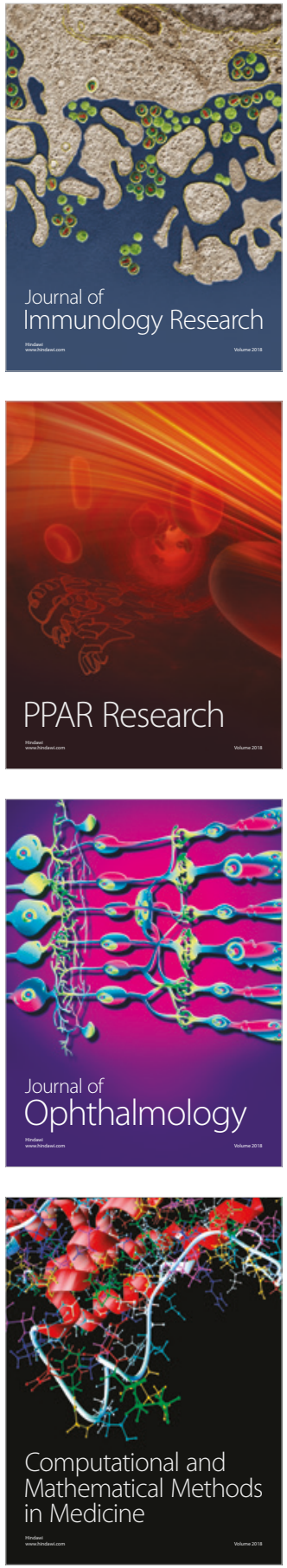

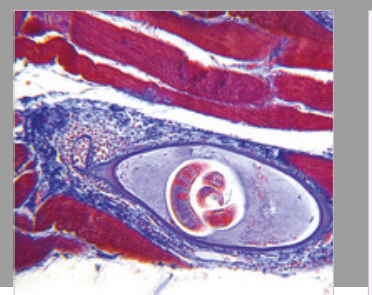

Gastroenterology Research and Practice

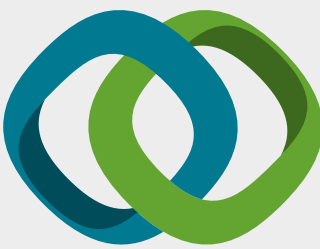

\section{Hindawi}

Submit your manuscripts at

www.hindawi.com
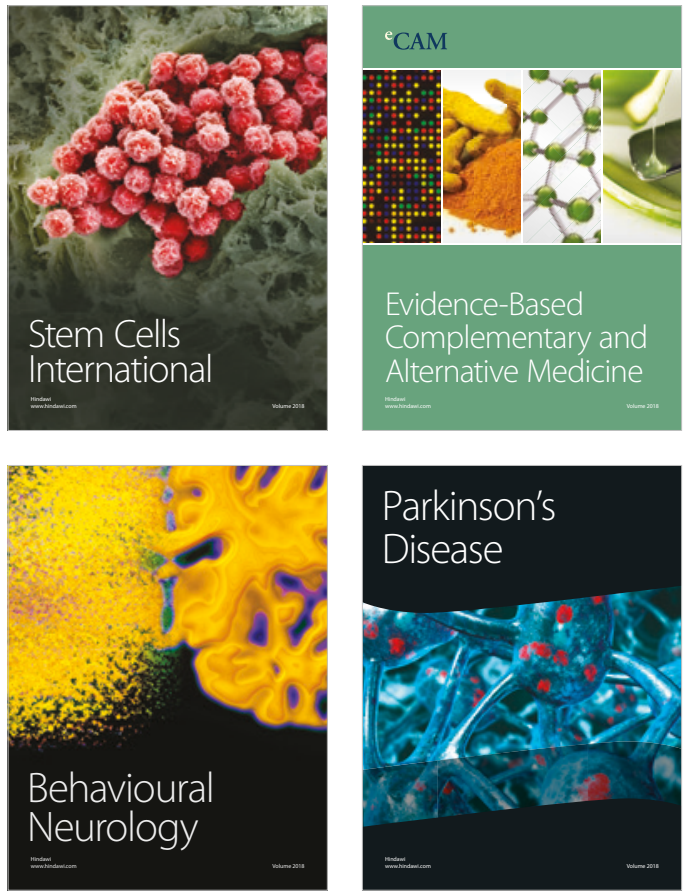

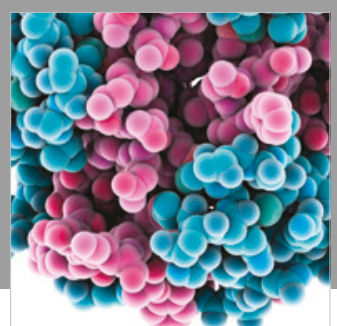

ournal of

Diabetes Research

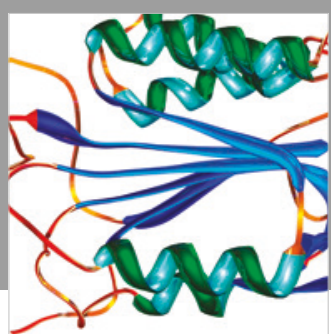

Disease Markers
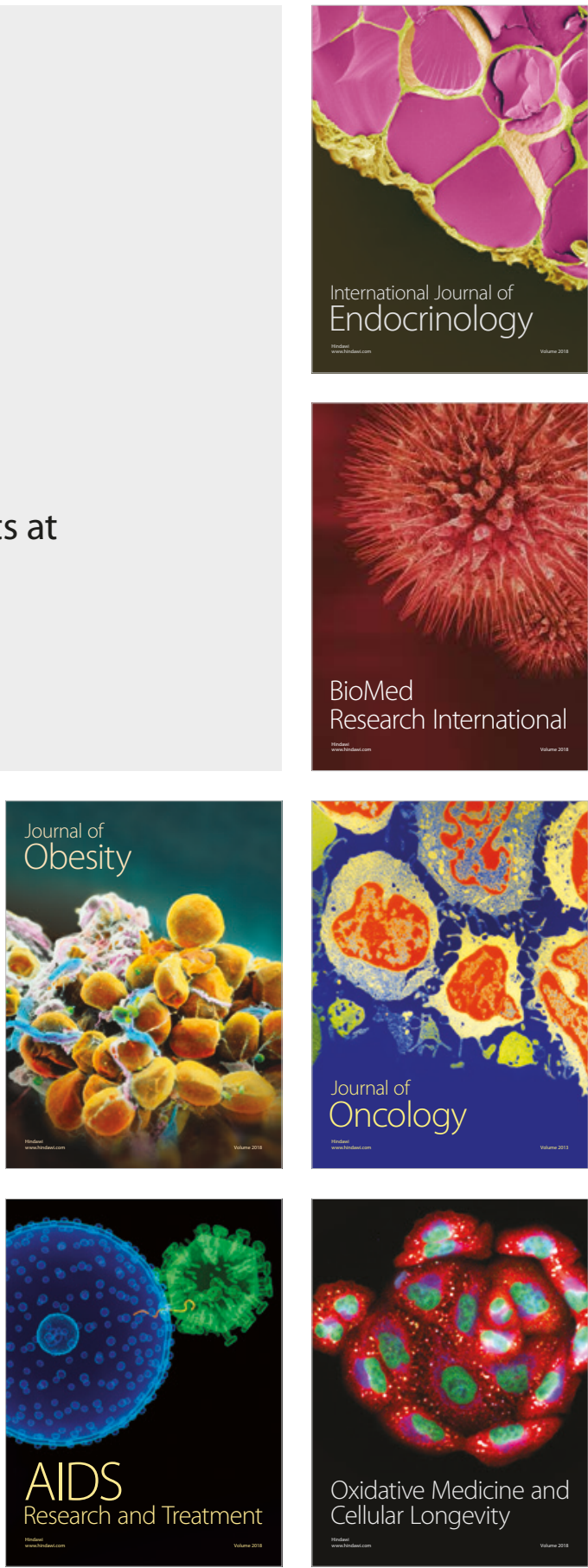